\title{
Perfectly Matched Layers for Frequency Domain Integral Equation Acoustic Scattering Problems
}

\author{
E.J. Alles, K.W.A. van Dongen \\ Laboratory of Acoustical Imaging and Sound Control \\ Department Imaging Science and Technology \\ Delft University of Technology, The Netherlands \\ Email: E.J.Alles@tudelft.nl, K.W.A.vanDongen@tudelft.nl
}

\begin{abstract}
Simulations of acoustic wavefields in inhomogeneous media are always performed on finite numerical domains. If contrasts in reality extend over the domain boundaries of the numerical volume, unwanted, unphysical reflections off the boundaries will occur. One technique to suppress these reflections is to attenuate them in a locally and reflectionless absorbing boundary layer enclosing the spatial computational domain, a Perfectly Matched Layer (PML). This technique is commonly applied in time-domain simulation methods like FEM and FDTD, but has not been applied to the integral equation method. In this paper, a PML formulation for the three-dimensional frequency domain integral equation based acoustic scattering problem is derived. Three-dimensional acoustic scattering configurations are used to test the PML formulation. The results demonstrate that strong attenuation (a factor of 200 in amplitude) of the scattered pressure field is achieved for thin layers with a thickness of less than a wavelength, and that the PMLs themselves are virtually reflectionless. In addition, it is shown that the integral equation method, both with and without PMLs, accurately reproduces pressure fields by comparing the obtained results with analytical solutions.
\end{abstract}

Index Terms-Acoustics, Integral equations, Diffraction, Reflection, Refraction, Scattering, Propagation

\section{INTRODUCTION}

Numerical acoustic simulations can roughly be divided into three categories. First, there are computationally efficient methods operating within the Born approximation, as used in e.g. the Field II software [1]. However, due to strong assumptions, the Born approximation yields incomplete and inaccurate results [2].

The second category is composed of time domain techniques which operate beyond the Born approximation. Examples are finite difference time domain (FDTD) [3] and finite element methods (FEM) [4]. Although these methods in general yield better results, as they include e.g. multiple scattering, they require a dense spatial sampling of up to 20 elements per wavelength, especially in the contrast regions, and thus large amounts of memory.

The third category consists of methods which simulate acoustic scattering in the frequency domain by solving the scatter integral equation [5], [6], [7] by means of an inversion scheme. If treated carefully, these methods require a significantly coarser grid than FEM or FDTD and hence a reduced memory load. One such approach will be used in the remainder of this paper.

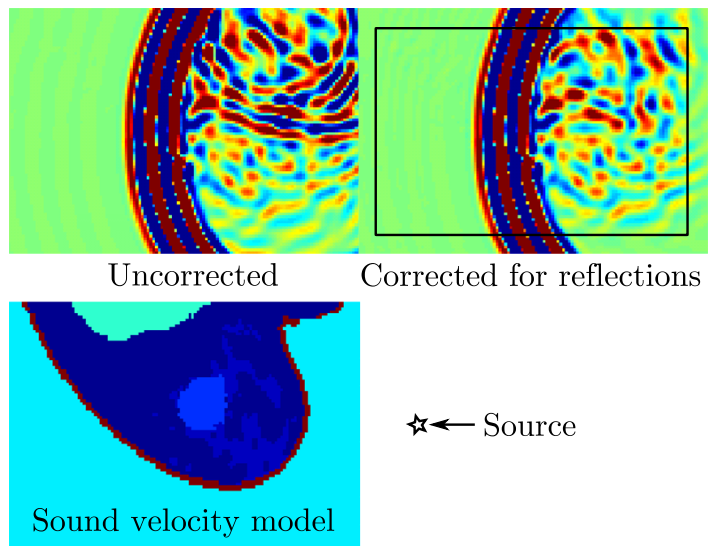

Fig. 1. Time frames of a wave field propagating in a three-dimensional breast model. The bottom left figure shows the sound velocity map of the corresponding slice. The velocities range, from blue to red, from 1478-1610 $\mathrm{m} / \mathrm{s}$ and the background medium is water $(c=1524 \mathrm{~m} / \mathrm{s})$. The source is located outside the numerical domain. The top left figure shows the wave field, including the unwanted additional reflections off the domain boundaries. In the top right figure the spurious reflections are suppressed by a thin absorbing layer enclosing the domain. The inner boundary of this layer is indicated by the black line. Color version can be found online. Media-color 1

The spatial numerical domain is required to be finite due to hardware restrictions. This requirement, however, introduces a significant problem if the acoustical contrast extends over the boundary of the spatial numerical domain. In these situations, the acoustic waves propagating through the contrast towards the domain boundary will experience a sharp change in contrast at the edge of the numerical domain. This leads to additional, unwanted reflections off the boundaries in the simulated wave fields, which need to be removed or suppressed.

This problem is clearly visible in Figure 1. Both top figures show the same time frame of the wave field propagating through a three-dimensional breast model with water as background medium, see bottom left figure. For both cases the volume is insonified by a Gaussian ultrasonic pulse which propagates through the breast and is scattered by the inhomogeneities in the breast. The simulations are based on the scatter integral equation discussed later on in this paper.

In the top left figure, in addition to the scattered field a reflection off the domain boundary is clearly visible. This reflection is due to the finiteness of the spatial numerical domain. In the top right figure, this reflection is suppressed 
and only scattering due to contrast inside the spatial numerical domain is modelled. Note that inhomogeneities outside the numerical domain cannot be taken into account using a finite domain, regardless of which simulation technique is used.

In order to suppress the unwanted reflections off the spatial domain boundaries, several techniques have been devised. The conceptually easiest technique is tapering: gradually reducing the contrast functions towards the domain boundaries. If this reduction is gradual enough with respect to wavelength, no reflection will occur as no discontinuities are present. Unfortunately, this requires thick layers which significantly increase the memory load and the computational time.

A different technique, commonly found in FDTD, is based on the absorbing boundary condition (ABC) [8]. A major problem with this technique, however, is that the attenuation is strongly angle dependent. Consequently, the boundary is not reflectionless for most angles.

Instead of using tapering or ABCs, in this work we will research the application of boundary layers with absorbing (material) properties to the integral equation method. This method has the advantages that the angular dependence problem of the $\mathrm{ABC}$ is reduced and, if applied properly, a significantly thinner layer as compared to tapering can be used. However, care has to be taken that no reflections occur off the boundaries of the absorbing layer instead. A technique with strong attenuation and guaranteed reflectionless absorption is the Perfectly Matched Layer (PML). This technique was originally developed for two dimensional electromagnetic wave problems [9] and later on expanded to three dimensional problems [10], [11]. Others have applied the method to elastodynamic wave fields [12], [13], [14] and acoustic wave fields [15], [16].

To the best of our knowledge, the PML formulation has never been applied to integral equation methods for acoustic scattering problems. In this paper we derive a PML formulation for the integral equation and implement this to show its effectiveness for acoustic scattering integral equation problems.

Note that PMLs are not applied to account for the scattering occurring outside the spatial numerical domain, but only to allow for truncation of the domain without introducing additional, unwanted reflections. Truncation of the spatial numerical domain will omit all the acoustic contrast outside the numerical domain and hence yield incomplete results.

\section{THEORY}

Before we derive a PML formulation of the scatter integral equation, first the non-PML version will be discussed [5], followed by the PML theory based on [17]. This theory is then applied to the scatter integral equation.

In figure 2 the various spatial domains used throughout this paper are defined. Let $\vec{r}$ denote a vector in the infinitely extended spatial domain $\mathbb{D}^{\text {tot }}$. Domain $\mathbb{D}$ is the domain of interest, $\mathbb{D}^{\mathrm{PML}}$ the PML domain enclosing $\mathbb{D}$ and $\mathbb{D}^{\text {num }}$ is the union of $\mathbb{D}$ and $\mathbb{D}^{\mathrm{PML}}$, which is the complete numerical spatial domain in which acoustic wavefields are modelled. Scattering occurs due to the presence of a contrast in the region $\mathbb{D}^{\text {scat }}$. The outer and inner boundaries of the numerical and the PML domain are $\partial \mathbb{D}^{\text {num }}$ and $\partial \mathbb{D}^{\text {PML }}$, respectively.

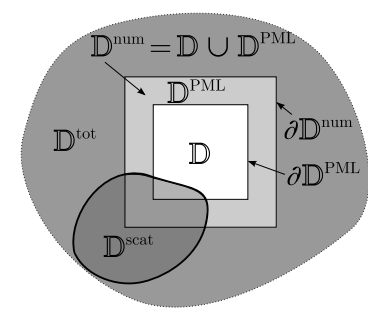

Fig. 2. The unbounded acoustic scattering domain $\mathbb{D}^{\text {tot }}$ with the spatial domains $\mathbb{D}, \mathbb{D}^{\mathrm{PML}}, \mathbb{D}^{\text {num }}, \mathbb{D}^{\text {scat }}, \partial \mathbb{D}^{\text {num }}$, and $\partial \mathbb{D}^{\mathrm{PML}}$.

In the remainder of this document the symbol ^ indicates that quantities are defined in the temporal Fourier domain.

\section{A. Scattering Theory}

Consider a volume $\mathbb{D}^{\text {tot }}$ with homogeneous acoustic material properties. In the absence of contrast, the actual pressure wave field $\hat{p}^{\text {tot }}(\vec{r})$ is referred to as the incident pressure wave field $\hat{p}^{\text {inc }}(\vec{r})$. However, if on top of the homogeneous background medium inhomogeneities are present, scattering will occur and the total field equals

$$
\hat{p}^{\text {tot }}(\vec{r})=\hat{p}^{\text {inc }}(\vec{r})+\hat{p}^{\text {scat }}(\vec{r}),
$$

where the difference between the total and incident pressure wave field is referred to as the scattered wave field $\hat{p}^{\text {scat }}(\vec{r})$.

It can be shown (e.g. [5]) that $\hat{p}^{\text {scat }}(\vec{r})$ satisfies the inhomogeneous Helmholtz equation

$$
\nabla^{2} \hat{p}^{\text {scat }}(\vec{r})+\hat{k}^{2} \hat{p}^{\text {scat }}(\vec{r})=-\hat{S}(\vec{r})
$$

with

$$
\hat{S}(\vec{r})=\left\{\begin{array}{cc}
\hat{k}^{2} X^{\kappa}(\vec{r}) \hat{p}^{\operatorname{tot}}(\vec{r}) & \forall \vec{r} \in \mathbb{D}^{\text {scat }} \\
+\vec{\nabla} \cdot\left[X^{\rho}(\vec{r}) \vec{\nabla} \hat{p}^{\operatorname{tot}}(\vec{r})\right] & \\
0 & \text { otherwise }
\end{array}\right.
$$

where $\vec{\nabla}$ indicates the gradient operator, - indicates an inner product, $\hat{k}$ is the wavenumber in the background medium, $X^{\kappa}(\vec{r})=\frac{\kappa^{\mathrm{scat}}(\vec{r})-\kappa^{\mathrm{bg}}}{\kappa^{\mathrm{bg}}}$ is the contrast in compressibility $\kappa$, and $X^{\rho}(\vec{r})=\frac{\rho^{\mathrm{bg}}-\rho^{\text {scat }}(\vec{r})}{\rho^{\text {scat }}(\vec{r})}$ is the contrast in volume density of mass $\rho$. Superscript bg indicates the background medium, scat the additional inhomogeneities.

A solution for Equation (2-3) is obtained by convolving $\hat{S}(\vec{r})$ with $\hat{G}\left(\vec{r}, \vec{r}^{\prime}\right)$, the Green's function for the homogeneous background medium. This function is the solution of the Helmholtz equation for a three-dimensional Dirac source distribution and satisfies the radiation condition. Using Equation (1) this yields

$$
\hat{p}^{\text {tot }}(\vec{r})=\hat{p}^{\text {inc }}(\vec{r})+\left[\hat{G} *_{\mathbb{D}^{\text {tot }}} \hat{S}\right](\vec{r}),
$$

in which $*_{\mathbb{D}^{\text {tot }}}$ is a spatial convolution over $\mathbb{D}^{\text {tot }}$. Due to the appearance of $\hat{p}^{\text {tot }}(\vec{r})$ in the contrast source term $\hat{S}(\vec{r})$, Equation (4) represents an integral equation of the second kind for known $\hat{p}^{\text {inc }}(\vec{r}), X^{\kappa}(\vec{r})$ and $X^{\rho}(\vec{r})$ and unknown $\hat{p}^{\text {tot }}(\vec{r})$. In literature this is referred to as the forward problem.

Problems arise when the spatial domain is limited to the finite numerical domain $\mathbb{D}^{\text {num }}$, while the contrast extends 
to regions outside $\mathbb{D}^{\text {num }}$, as additional, unwanted reflections originating from the boundary $\partial \mathbb{D}^{\text {num }} \cap \mathbb{D}^{\text {scat }}$ appear in the results. These additional reflections will be suppressed by applying PMLs as discussed in the next subsection.

\section{B. PML Theory}

The previous theory was given for real coordinates $\vec{r}$. From a mathematical point of view, however, nothing restricts $\vec{r}$ to be real, and it turns out to be advantageous to allow for complex coordinates, a choice which automatically leads to PMLs. A good overview of the theory on PMLs is presented in [17].

To explain the theory behind PMLs, we start with the onedimensional homogeneous Helmholtz equation

$$
\frac{\partial^{2}}{\partial x^{2}} \hat{f}(x)+\hat{k}^{2} \hat{f}(x)=0
$$

with plane wave solutions

$$
\hat{f}(x)=e^{i(\hat{k} x-\omega t)}
$$

for real $x$. These solutions are analytic functions.

By allowing $\tilde{x}=x+i h(x)$ to become complex, the so-called analytically continued one-dimensional Helmholtz equation

$$
\frac{\partial^{2}}{\partial \tilde{x}^{2}} \hat{g}(\tilde{x})+\hat{k}^{2} \hat{g}(\tilde{x})=0
$$

is obtained which has as solution

$$
\hat{g}(\tilde{x})=e^{i(\hat{k} \tilde{x}-\omega t)}=e^{-\hat{k} h(x)} e^{i(\hat{k} x-\omega t)} .
$$

Note that for real $\hat{k} h(x)>0$ this function is exponentially decaying.

An important observation is that the unicity [18] of the analytic continuation guarantees that solving for $\hat{g}(\tilde{x})$ on any domain where $h(x)=0$ yields $\hat{f}(x)$. This guarantees that $\hat{g}(\tilde{x})$ is only absorbed where $\hat{k} h(x)>0$. Consequently, a convenient choice for $\hat{k} h(x)$ is

$$
\hat{k} h(x) \begin{cases}>0 & \forall x \in \mathbb{D}^{\mathrm{PML}} \\ =0 & \forall x \in \mathbb{D}\end{cases}
$$

In this way, $\hat{g}(x)$ is equal to $\hat{f}(x)$ for $x \in \mathbb{D}$ and exponentially decaying for $x \in \mathbb{D}^{\mathrm{PML}}$. Thus, one can arrange for a finite layer with absorbing properties which itself is reflectionless as plane waves propagating from $\mathbb{D}$ to $\mathbb{D}^{\mathrm{PML}}$ are not reflected back to $\mathbb{D}$; a PML is obtained.

Analytical continuation of the Helmholtz equation from $x$ to $\tilde{x}=x+i h(x)$ where $h(x)$ is a real function led to local attenuation of propagating waves. However, for evanescent waves with imaginary wavenumber $\hat{k}=i \underline{\hat{k}}$, in which $\underline{\hat{k}}$ is positive real, the solution to the analytically continued Helmholtz equation reads

$$
\hat{g}(\tilde{x})=e^{i(\hat{k} \tilde{x}-\omega t)}=e^{-i \underline{\hat{k}} h(x)} e^{i(\hat{k} x-\omega t)} .
$$

Thus, a PML does not attenuate evanescent waves for real $h(x)$.

Even though evanscent waves are exponentially decaying without a PML, PMLs can be used to increase their decay rate by allowing $h(x)$ to become complex; $h(x)=l(x)-i m(x)$. Since $\tilde{x}=x+i h(x)=x+m(x)+i l(x)$, this results, for $m(x)>0$, in coordinate stretching which causes evanescent waves to experience a spatial extent larger than it actually is, and hence an increase in decay per unit distance. Hence, the solution to the analytically continued Helmholtz equation is

$$
\hat{g}(\tilde{x})=e^{-i \underline{\hat{k}} l(x)} e^{-\underline{\hat{k}} m(x)} e^{i(\hat{k} x-\omega t)},
$$

(for imaginary wavenumber $\hat{k}=i \underline{\hat{k}}$ ) and for propagating waves

$$
\hat{g}(\tilde{x})=e^{-\hat{k} l(x)} e^{i\left(\hat{k}^{\prime} x-\omega t\right)},
$$

with real valued wavenumber $\hat{k}^{\prime}=\left(1+\frac{m(x)}{x}\right) \hat{k}$. Thus, for positive $l(x)$ and $m(x)$ both evanescent and propagating waves are damped in the PML.

Unfortunately, coordinate stretching leads to an effective wavenumber $\hat{k}^{\prime}>\hat{k}$ and thus to waves of shorter wavelength, which are less accurately represented on the discrete grid. Due to this loss of accuracy, coordinate stretching will cause minor reflections off the PMLs as explained in the next subsection.

\section{PML Implementation}

Solving integral equations along complex contours is less convenient than along real contours. Therefore, it is desirable to rewrite Equation (7) back to real coordinates. Starting with $\tilde{x}=x+i h(x)$ and setting $\frac{\partial h(x)}{\partial x}=\frac{\sigma(x)}{\omega}$ with $\sigma(x) \neq i \omega$, the spatial derivative with respect to $\tilde{x}$ can be written as

$$
\frac{\partial}{\partial \tilde{x}}=\frac{1}{1+i \frac{\partial h(x)}{\partial x}} \frac{\partial}{\partial x}=\frac{1}{1+i \frac{\sigma(x)}{\omega}} \frac{\partial}{\partial x}=\hat{X}^{\sigma}(x) \frac{\partial}{\partial x} .
$$

Note that $\sigma(x)=i \omega$ is a particular case of pure coordinate stretching that would not attenuate propagating waves. For media that are $x$-invariant in $\mathbb{D}^{\mathrm{PML}}$, the above substitution is the only alteration necessary to yield a Helmholtz equation on real coordinates. Hence, from here on we assume the medium to be invariant in the direction the PML works on. The division by angular frequency $\omega$ appears in order to obtain frequencyindependent attenuation.

Applying the above substitution to the one-dimensional homogeneous Helmholtz equation (7) yields, for $\omega \neq 0$,

$$
\frac{\partial^{2} \hat{g}(x)}{\partial x^{2}}+\hat{k}^{\prime \prime 2}(x) \hat{g}(x)=-\frac{1}{\hat{X}^{\sigma}(x)} \frac{\partial \hat{X}^{\sigma}(x)}{\partial x} \frac{\partial \hat{g}(x)}{\partial x}
$$

where the appearance of a complex wavenumber $\hat{k}^{\prime \prime}(x)=$ $\left(1+i \frac{\sigma(x)}{\omega}\right) \hat{k}$ is equivalent to assuming a locally complexvalued, lossy medium. The source term on the right-hand side of Equation (14) appears to cancel out any reflections of waves propagating from real to complex media. Note that the amplitude of this source is frequency dependent and increases for decreasing frequency.

In three dimensions, the PML has to be formulated separately for each component of the gradient operator. Thus, contrast function $\hat{X}^{\sigma}$ becomes a diagonal matrix with diagonal elements $\hat{X}_{x}^{\sigma}(x), \hat{X}_{y}^{\sigma}(y)$ and $\hat{X}_{z}^{\sigma}(z)$. Consequently, the gradient operator is replaced by

$$
\left(\begin{array}{ccc}
\hat{X}_{x}^{\sigma}(x) & 0 & 0 \\
0 & \hat{X}_{y}^{\sigma}(y) & 0 \\
0 & 0 & \hat{X}_{z}^{\sigma}(z)
\end{array}\right)\left(\begin{array}{c}
\frac{\partial}{\partial x} \\
\frac{\partial}{\partial y} \\
\frac{\partial}{\partial z}
\end{array}\right)=\hat{X}^{\sigma}(\vec{r}) \vec{\nabla} .
$$




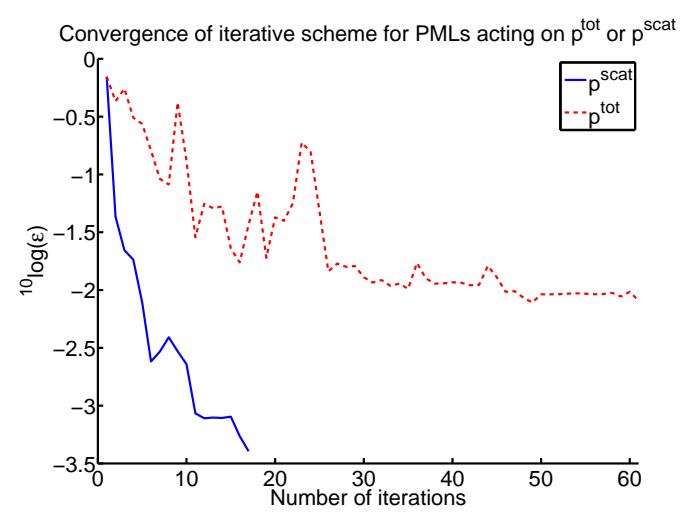

Fig. 3. Convergence of the iterative solver of three-dimensional simulations with PMLs acting on $\hat{p}^{\text {scat }}$ or $\hat{p}^{\text {tot }}$ enclosing the numerical volume. The simulated situation is discussed in section IV-B, the source transmitted a continuous wave at $1.5 \mathrm{MHz}$. The normalized $L_{2}$-norm of the residual $\epsilon$ is a measure of how far the iterative solver is from solving the system. It is obvious that the situation where only $\hat{p}^{\text {scat }}$ is attenuated requires far less iterations than when $\hat{p}^{\text {tot }}$ is attenuated. Color version can be found online. Media-color 3

The latter shorthand notation will be used throughout the rest of this paper for better readability.

\section{PML Formulation of the scatter integral equation}

Two different PML formulations for the scatter integral equation can be derived, one suppressing only the scattered field $\hat{p}^{\text {scat }}(\vec{r})$ inside the PML and one where the total field $\hat{p}^{\text {tot }}(\vec{r})$ is suppressed. The latter approach seems more natural since the PML operates as a regular absorbing layer, whereas in the former case only selective absorption is achieved.

However, the scatter integral equation (4) is inverted by means of an iterative scheme as explained later on. This implies that if the total field, and hence the incident field, is attenuated inside the PML, the iterative scheme also has to account for the change in incident field. If instead only the scattered field is attenuated, the incident field remains unchanged and therefore a faster convergence of the iterative solver is expected. That this is indeed the case is shown in Figure 3, from which it is clear that the difference in convergence rate is significant. In this figure, $\epsilon$, the $L_{2}$-norm of the residual normalized by the $L_{2}$-norm of the known incident field, is shown as a function of the number of iterations.

Furthermore, in acoustical simulations it is sufficient to only attenuate the scattered field, as this is the only component of the total field containing the erroneous reflections. Since simulations with PMLs acting on only $\hat{p}^{\text {scat }}$ have a much higher convergence rate, only this case will be studied.

A PML formulation for the inhomogeneous Helmholtz equation (2-3) is obtained by $(i)$ analytically continuing Equation (2-3) by using Equation (15), (ii) rewriting the result into an inhomogeneous Helmholtz equation, (iii) convolving the source term with the Green's function and (iv) using Equation (1). In this way we obtain a PML corrected integral equation which reads

$$
\begin{aligned}
& \hat{p}^{\text {inc }}(\vec{r})-\left[\hat{G} *_{\mathbb{D}^{\text {num }}} \hat{S}^{\text {inc }}\right](\vec{r})= \\
& \hat{p}^{\text {tot }}(\vec{r})-\left[\hat{G} *_{\mathbb{D}^{\text {num }}} \hat{S}^{\text {tot }}\right](\vec{r}),
\end{aligned}
$$

where

$$
\hat{S}^{\text {inc }}(\vec{r})=\hat{X}^{\sigma}(\vec{r}) \vec{\nabla} \cdot\left[\hat{X}^{\sigma}(\vec{r}) \vec{\nabla} \hat{p}^{\text {inc }}(\vec{r})\right]-\nabla^{2} \hat{p}^{\text {inc }}(\vec{r})
$$

and

$$
\begin{aligned}
\hat{S}^{\text {tot }}(\vec{r}) & =\hat{k}^{2} X^{\kappa}(\vec{r}) \hat{p}^{\text {tot }}(\vec{r})-\nabla^{2} \hat{p}^{\text {tot }}(\vec{r}) \\
& +\hat{X}^{\sigma}(\vec{r}) \vec{\nabla} \cdot\left[\hat{X}^{\sigma}(\vec{r})\left(X^{\rho}(\vec{r})+1\right) \vec{\nabla} \hat{p}^{\text {tot }}(\vec{r})\right] .
\end{aligned}
$$

Note that the incident field is corrected by an additional source term $\hat{S}^{\mathrm{inc}}(\vec{r})$, but that the right hand side still only contains one convolution. As the incident field is corrected before the iterative scheme commences, this means that the computational load per iteration is not significantly increased as compared to the non-PML version.

The contrasts in compressibility and density are required to be spatially invariant inside $\mathbb{D}^{P M L}$ in the direction the PML acts on. Spatial invariance in the $x$-direction is obtained by setting $X^{\kappa}\left(x \in \mathbb{D}^{\mathrm{PML}}, y, z\right)$ to $X^{\kappa}\left(x \in \partial \mathbb{D}^{\mathrm{PML}}, y, z\right)$ and $X^{\rho}\left(x \in \mathbb{D}^{\mathrm{PML}}, y, z\right)$ to $X^{\rho}\left(x \in \partial \mathbb{D}^{\mathrm{PML}}, y, z\right)$. Invariance in the $y$ - and $z$-directions are obtained in an identical way.

\section{IMPLEMENTATION}

For known incident field and contrasts, integral Equation (1618 ) is solved on $\mathbb{D}^{\text {num }}$ for unknown $\hat{p}^{\text {tot }}(\vec{r})$ by inverting the integral equation. As analytic inversion is not possible, the problem will be solved numerically on a discretized grid.

After discretization, the resulting integral Equation (16-18) can be represented as a matrix-vector equation of the form

$$
A p^{\text {tot }}=\mathrm{p}^{\text {inc }}
$$

where $\mathrm{p}^{\text {inc }}$ is the vector containing the known values of $\hat{p}^{\text {inc }}(\vec{r})-\left[\hat{G} *_{\mathbb{D}^{\text {num }}} \hat{S}^{\text {inc }}\right](\vec{r})$ on the points in the discrete grid, $\mathrm{p}^{\text {tot }}$ is the vector containing the unknown values of $\hat{p}^{\text {tot }}(\vec{r})$ on the grid and $A$ is the known matrix governing the integral equation including the PMLs.

Direct inversion of this matrix is not possible due to memory and computation time restrictions. Therefore, the integral equation will be solved iteratively using a Bi-CGSTAB scheme [19] because of its fast convergence and simplicity.

Discretization of Equation (16-18) is not trivial. Firstly, to overcome problems associated with the singularity of the Greens function we use its weak form [20]. Secondly, spatial derivatives have to be taken of discontinuous functions, and therefore cannot be taken in the spatial Laplace domain. To improve on accuracy, rather than the common symmetric threepoint stencil, a symmetric 17-point stencil is used. Thirdly, the spatial convolution is a computationally expensive operator. To reduce the computational load, the convolution is computed in the spatial Fourier domain using FFTs, at the expense of an increase in memory load.

Unfortunately, PMLs are only guaranteed to be reflectionless in the analytical case. Thus, the source term in equation 
(14) can only completely cancel out the reflections caused by a space-variant $\hat{k}^{\prime \prime}(x)$ if it is analytically convolved with the Green's function. However, on a discrete grid the convolution and the Green's function are only approximations, hence minor reflections off the PML for discontinuous $\sigma(x)$ are expected.

\section{EXPERIMENTS}

In order to investigate the performance of the PML we will first test the accuracy of the method, followed by tests on the effectiveness of PMLs, both in terms of achieved attenuation and reflectionlessness, and by experiments on the convergence of the iterative scheme. Finally the effects of allowing for a complex or smooth PML contrast function $\sigma(\vec{r})$ are studied.

\section{A. Validation of the Integral Equation Method}

The simplest scattering problem for which an analytical solution exists [21] is the one-dimensional case of a plane wave reflecting off a single interface between the background medium and the contrast at normal incidence.

To validate the integral equation method, both with and without PML, a three-dimensional volume of $1024 \mu \mathrm{m} \times$ $1024 \mu \mathrm{m} \times 1024 \mu \mathrm{m}$ of blood $\left(\rho^{\mathrm{bg}}=1050 \mathrm{~kg} \mathrm{~m}^{-3}\right.$, $\left.\kappa^{\mathrm{bg}}=3.91 \cdot 10^{-10} \mathrm{~Pa}^{-1}\right)$ is simulated using elements of size $8 \mu \mathrm{m} \times 8 \mu \mathrm{m} \times 8 \mu \mathrm{m}$. Half of the volume, starting at $x_{0}=512 \mu \mathrm{m}$, is modelled to mimic fat $\left(\rho^{\text {scat }}=960 \mathrm{~kg} \mathrm{~m}^{-3}\right.$, $\left.\kappa^{\text {scat }}=4.82 \cdot 10^{-10} \mathrm{~Pa}^{-1}\right)$. The transient plane wave has an amplitude $A_{\mathrm{i}}=0.25 \mathrm{MPa}$, a center frequency of $20 \mathrm{MHz}$, a bandwidth of $83 \%$, and has zero phase at $x=0 \mathrm{~m}$. The point of observation is $\vec{r}_{\mathrm{obs}}=(375 \mu \mathrm{m}, 512 \mu \mathrm{m}, 512 \mu \mathrm{m})$.

The resulting total fields $p^{\text {tot }}\left(\vec{r}_{\text {obs }}\right)$ for the analytic case, and from simulations with and without PML are shown in Figure 4. Both the PML and non-PML simulations accurately reproduce the amplitudes and travel times of the incident pulse and the reflection off the contrast interface. The nonPML result contains additional reflections off the domain boundaries, which are effectively suppressed in the result obtained by simulations including PMLs.

The integral equation method, both in- and excluding PMLs, is also tested against the three-dimensional analytic solution for a plane wave scattering off a soft homogeneous spherical contrast [22]. As in the situation above, a volume of $1024 \mu \mathrm{m} \times$ $1024 \mu \mathrm{m} \times 1024 \mu \mathrm{m}$ of blood is simulated. In this case, a sphere of radius $256 \mu \mathrm{m}$ mimicking fat is located centrally in the cube, and the volume is divided in elements of $4 \mu \mathrm{m} \times$ $4 \mu \mathrm{m} \times 4 \mu \mathrm{m}$.

In Figure 5, the analytic solution is shown together with the solution obtained by solving the scatter integral equation for the complete sphere. The incident field propagates, in this figure, from left to right with increasing time. The two solutions show excellent agreement.

In addition, in this figure the case of a truncated sphere is shown, first for the case where the contrast is simply cut off, i.e. without applying a PML. Although the early time slice at $t=0.74 \mu$ s agrees with the full and analytic solutions, it is clear from the slice at $t=1.04 \mu$ s that truncating the contrast introduces a reflection off the domain boundary.

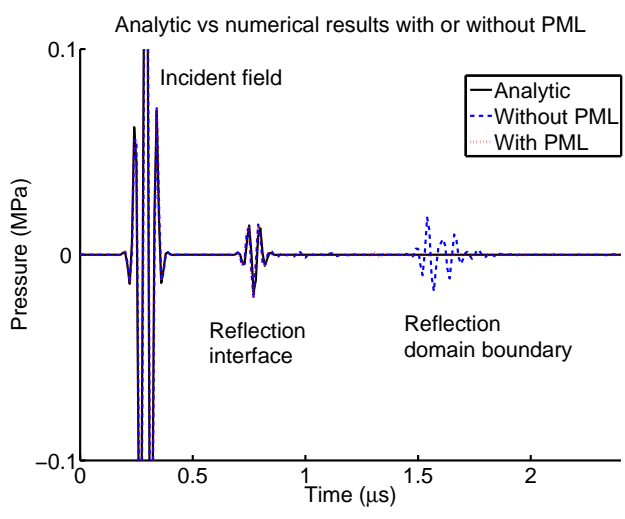

Fig. 4. Comparison of the analytical solution to the scatter problem for plane waves reflecting under normal incidence with simulations with and without PMLs. To improve clarity,the pressure axis is clipped. Both simulations reproduce accurate amplitudes and travel times of both the incident field and the reflection off the interface, but the non-PML result shows additional reflections off the domain boundaries which are effectively suppressed in the PML result. Color version can be found online. Media-color 4

In the bottom row of Figure 5, time slices of the truncated situation, including a PML of a thickness of approximately 3.3 wavelengths, are shown. From these images it is clear that the PML suppresses the reflection off the domain boundary while introducing only a very weak reflection off the PML itself. Furthermore, the scattering occuring off the modelled contrast is in agreement with the analytical solution of the full problem. As the distal half of the sphere is absent, naturally this half does not contribute to the total pressure field.

\section{B. PML Effectiveness}

To test the effectiveness of the PML formulation in Equation (16-18), we consider the situation sketched in Figure 6. It consists of a cubic contrast of $160 \mu \mathrm{m}$ in all three directions centered in a volume of dimensions $512 \mu \mathrm{m} \times 512 \mu \mathrm{m} \times$ $512 \mu \mathrm{m}$. Again blood is chosen as homogeneous background medium, while the contrast is modelled to mimic fat.

Outside the modelled cube a transducer with radiating surface of area $400 \mu \mathrm{m} \times 27 \mu \mathrm{m}$ is placed to generate an incident pressure field at a frequency of $10 \mathrm{MHz}$. The spatial domain is divided into $64^{3}$ cubic elements of $8 \mu \mathrm{m} \times 8$ $\mu \mathrm{m} \times 8 \mu \mathrm{m}$ each. The same discretization is used throughout the remaining part of this section.

Note that in this situation there would be no need for PMLs as no contrast is present at any of the domain boundaries. However, this situation allows for easy determination of incident, scattered and total fields and thus easy quantification of the attenuation achieved in the scattered field by applying PMLs.

For simplicity, in this situation the PML contrast function $\sigma(\vec{r})$ is chosen to be a step function, i.e.

$$
\sigma(\vec{r})=\left\{\begin{array}{ccl}
\text { constant } & \forall & \vec{r} \in \mathbb{D}^{\mathrm{PML}} \\
0 & \forall & \vec{r} \in \mathbb{D} .
\end{array}\right.
$$

The PML thickness $d$ is equal for all three dimensions as indicated in Figure 6. More elaborate PML contrast functions will be studied lateron. 

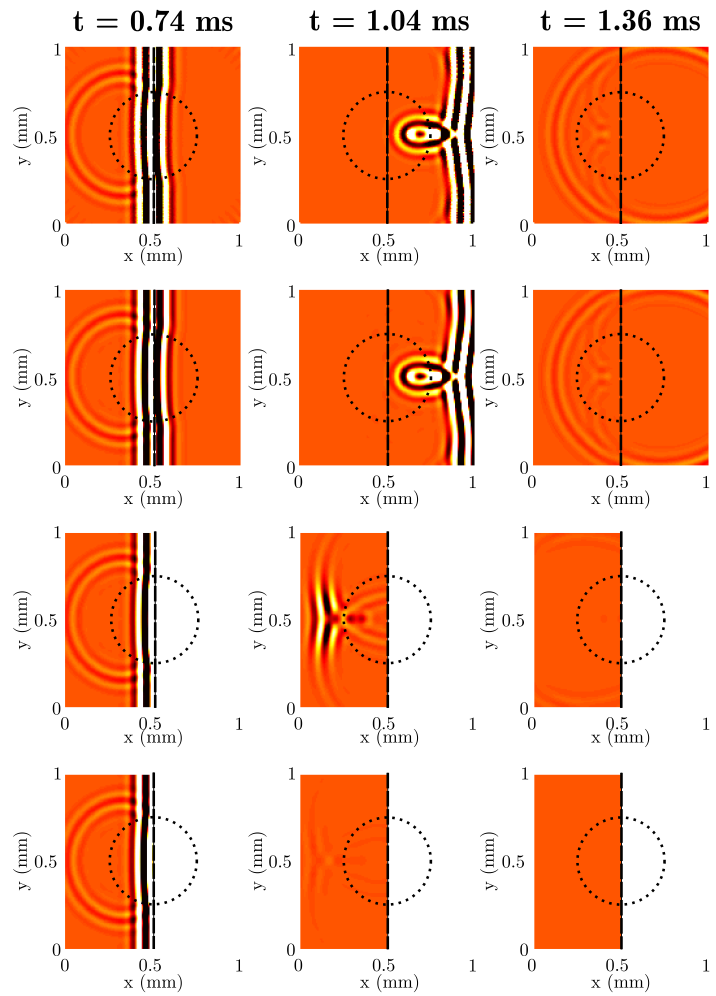

Fig. 5. Timeslices of the analytic solution (top row) of a plane wave scattering off a soft homogeneous sphere, together with the solution obtained by solving the scatter integral equation described in this work (second row). Observe that the two results show excellent agreement, both in amplitude and phase. In the bottom two rows, the cubic volume is cut in half, thereby truncating the spherical contrast. If no PML is applied, a strong reflection off the domain boundary is observed (third row from the top), demonstrating the need for careful treatment of contrast truncation. Observe that the scattering present in the first timeslice agrees with the analytic solution for the full sphere. If a PML is applied (bottom row), the unphysical reflection off the domain boundary is removed and only a very weak reflection off the PML is introduced. All twelve images use the same colorbar which has been clipped by a factor of ten to increase visibility of the scattered fields. High brightness means high pressure. The dotted circles indicate the location of the sphere, and the dashed lines indicate where, in the bottom two rows, domain $\mathbb{D}$ has been truncated. All images shown are at a height of $512 \mu \mathrm{m}$, and the incident plane wave propagates from left to right with increasing time. Media-color 5

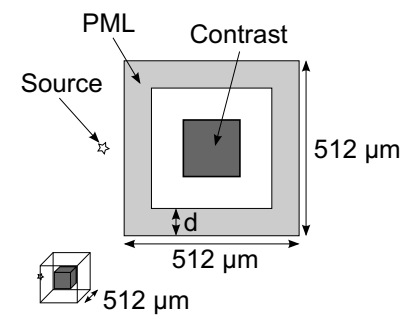

Fig. 6. Sketch of the situation used to evaluate the PML effectiveness.

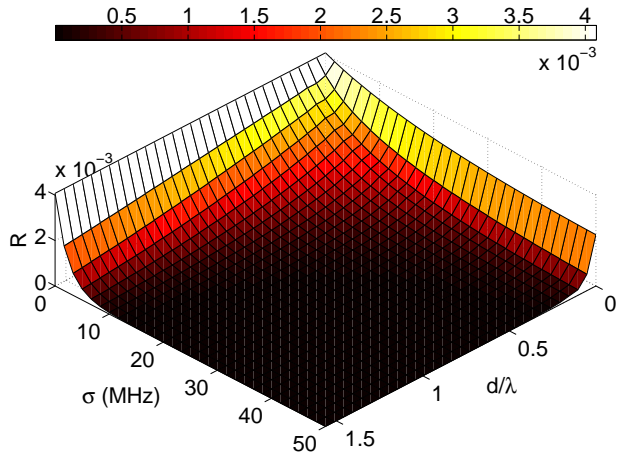

Fig. 7. Normalized sum of the remaining scatter field $\hat{p}^{\text {scat }}$ at $\partial \mathbb{D}^{\text {num }}$ as a function of $\frac{d}{\lambda}$ and $\sigma$ after applying a PML. Simulations are performed at a frequency of $10 \mathrm{MHz}$ in the domain depicted in Figure 6. Color version can be found online. Media-color 7

Ideally, a PML attenuates strongly enough so that pressure fields scattered off the contrast block are fully attenuated before reaching $\partial \mathbb{D}^{\text {num }}$. In order to quantify the attenuation of the PML, the normalized sum $R\left(\frac{d}{\lambda}, \sigma\right)$ of the remaining scatter pressure field on $\partial \mathbb{D}^{\text {num }}$ is computed as a function of $\frac{d}{\lambda}$ and $\sigma$, i.e.

$$
R\left(\frac{d}{\lambda}, \sigma\right)=\frac{\sum_{\partial \mathbb{D}^{\text {num }}}\left|\mathrm{p}^{\text {scat }}\right|^{2}}{\sum_{\partial \mathbb{D}^{\text {num }}}\left|\mathrm{p}^{\text {inc }}\right|^{2}}
$$

The results are shown in Figure 7. It can be seen that increasing $d$ or $\sigma$ results in a smooth decrease of the scattered field. Also note the sharp change in $R$ starting directly at $\frac{d}{\lambda}=0$, indicating that even thin layers of much less than a wavelength thick result in strong attenuation. The remaining scatter field $\hat{p}^{\text {scat }}$ is decreased by a factor of more than 200 for large $\frac{d}{\lambda}$ and $\sigma$.

Next, it is tested whether the PMLs are indeed reflectionless. To this aim, we have used the situation sketched in Figure 8. It consists of the same cubic domain as in the previous experiment, though in this case no contrast is present and the source is placed at the center of the cube. Ideally, both with and without PMLs, the total field should equal the incident field as no contrast is present. So to quantify the reflectionlessness of the PML, the same sum of Equation (21) is used. However, in this case the summations run over $\mathbb{D}^{\text {num }}$ instead of just $\partial \mathbb{D}^{\text {num }}$, hence

$$
R\left(\frac{d}{\lambda}, \sigma\right)=\frac{\sum_{\mathbb{D}^{\text {num }}}\left|\mathrm{p}^{\text {scat }}\right|^{2}}{\sum_{\mathbb{D}^{\text {num }}}\left|\mathrm{p}^{\text {inc }}\right|^{2}} .
$$

Intuitively, it seems more logical to sum over $\mathbb{D}$ only, as it is of no relevance what the scattered pressure field inside the PML is. However, the size of $\mathbb{D}$ varies with $d$, so quantitative comparison between different situations is less straightforward due to normalization. Fortunately, experiments (not treated here) show that the amplitude of $\hat{p}^{\text {scat }}$ is roughly constant 


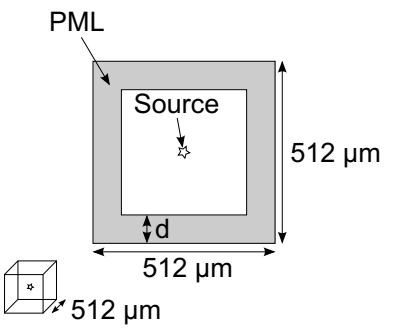

Fig. 8. Sketch of the situation used to evaluate PML reflectionlessness.

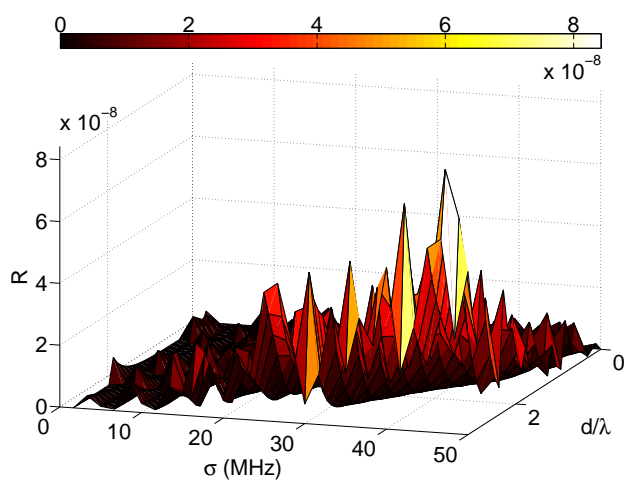

Fig. 9. Normalized sum of the PML-generated scatter field $\hat{p}^{\text {scat }}$ over $\mathbb{D}^{\text {num }}$ as a function of $\frac{d}{\lambda}$ and $\sigma$. Simulations are performed in the domain depicted in Figure 8 at a frequency of $20 \mathrm{MHz}$. Color version can be found online. Media-color 9

throughout $\mathbb{D}^{\text {num }}$, which means that the summations can be performed over $\mathbb{D}^{\text {num }}$ without problems.

The sum $R\left(\frac{d}{\lambda}, \sigma\right)$ is shown in Figure 9 for simulations performed at a frequency of $20 \mathrm{MHz}$. In general, $R$ increases with increasing $\frac{d}{\lambda}$ or $\sigma$ whereas for a reflectionless PML $R$ should equal 0 , indicating that more and more of the incident pressure field is scattered by the PML. However, the amplitude of the scattered pressure field generated by the PMLs is more than 100 times smaller than that of a field scattered off typical contrasts. Thus, the PMLs are virtually reflectionless.

\section{Convergence Iterative Scheme}

As can be observed from Equation (16-18), introducing PMLs results in adding contrasts to the problem under investigation. It is therefore expected that the convergence of the $\mathrm{Bi}$ CGSTAB scheme is decreased by incorporating the PMLs. To test the influence of the applied PML on the convergence of the iterative inversion scheme, the situation as depicted in Figure 6 is modelled.

In Figure 10 the number of iterations required to reach a maximum retaining error level $\epsilon_{\max }=1 \cdot 10^{-8}$ is plotted as a function of $\frac{d}{\lambda}$ and $\sigma$. This error level is defined as

$$
\epsilon=\frac{\sum_{\mathbb{D}_{\text {num }}}\left|p^{\text {inc }}-A p^{\text {tot }}\right|^{2}}{\sum_{\mathbb{D}^{\text {num }}}\left|p^{\text {inc }}\right|^{2}}
$$

In this case the problem was solved for a frequency of $20 \mathrm{MHz}$. It is clear from this figure that indeed the con-

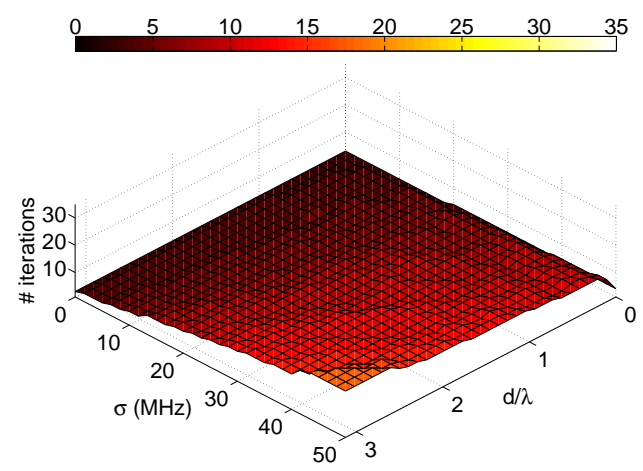

Fig. 10. Number of iterations required for various $\frac{d}{\lambda}$ and $\sigma$ at $20 \mathrm{MHz}$ to reach a normalized error $\epsilon=1 \cdot 10^{-8}$. Color version can be found online. Media-color 10

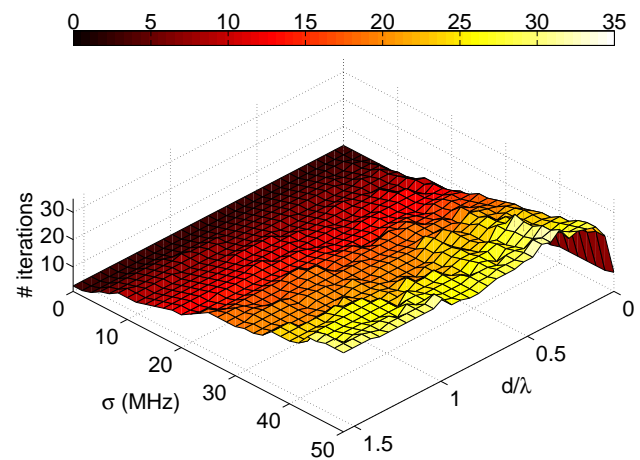

Fig. 11. Number of iterations required for various $\frac{d}{\lambda}$ and $\sigma$ at $10 \mathrm{MHz}$ to reach a normalized error $\epsilon=1 \cdot 10^{-8}$. Color version can be found online. Media-color 11

vergence rate decreases as more iterations are required for stronger or thicker PMLs. For this frequency, the number of iterations increases from 3 in the absence of PMLs to 21 for the strongest PML used in this experiment.

In Figure 11 the same experiment is repeated for a frequency of $10 \mathrm{MHz}$. Here we observe that for decreasing frequency the deterioration of the convergence rate due to PMLs increases. This effect was already predicted in subsection II-C.

An important observation to be made is that, especially for lower frequencies, the PML contrast amplitude $\sigma$ has a much greater impact on the convergence rate than the thickness $d$. This suggests that, in relation to convergence, thick PMLs are preferred over strong PMLs. This fits with the theory; in the case of an infinitely extended PML with zero strength no additional contrast is introduced $(\sigma=0)$ and yet no additional reflections will occur as no domain boundary is present.

\section{Smooth PML Contrast Function}

In subsection II-C it was shown that changing $\sigma(\vec{r})$ from the step function in Equation (20) to a smoother function would reduce the reflections off $\partial \mathbb{D}^{\mathrm{PML}}$. Even though in the above experiments these reflections were found to be negligibly small, i.e. more than 100 times smaller than fields scattered 


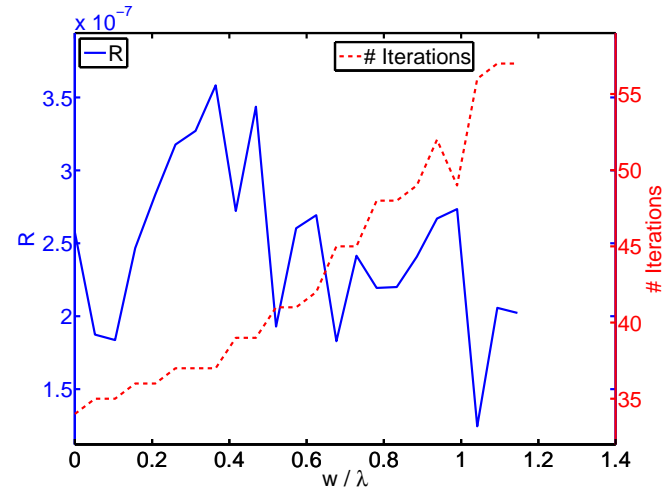

Fig. 12. Normalized sum of scatter pressure field $\hat{p}^{\text {scat }}$ over $\mathbb{D}^{\text {num }}$ and number of iterations required for various $w$. Simulations are performed over the domain depicted in Figure 8 at a frequency of $10 \mathrm{MHz}$. Only a marginal decrease in reflection off the PML is achieved by smoothing the PML contrast function, at the cost of a significant increase in computation time. Color version can be found online. Media-color 12

off typical contrasts, in this section we will attempt to further diminish them.

In order to diminish these reflections a smooth transition of the PML contrast function between $\mathbb{D}$ and $\mathbb{D}^{\mathrm{PML}}$ should be achieved. Therefore, in the one-dimensional case we multiply, in the transition region, $\sigma(x)$ of Equation (20) with a smoothly function $\phi\left(x^{\prime}\right)$ :

$$
\phi\left(x^{\prime}\right)=\frac{1}{2}-\frac{1}{2} \cos \left(\frac{\pi x^{\prime}}{w}\right), \quad 0 \leq x^{\prime} \leq w
$$

where $w$ is the width of the transition region.

To determine the effect of smoothing $\sigma$ on the reflectionlessness of the PML, the situation depicted in Figure 8 is used. At $10 \mathrm{MHz}$, a PML of fixed thickness $\frac{d}{\lambda}=1.56$ and PML contrast amplitude $\sigma=50 \mathrm{MHz}$ is multiplied with smoothing function $\phi\left(x^{\prime}\right)$ for varying $w$, and weighted to achieve similar attenuation for all $w$. The normalized sum of Equation (21) is computed over $\mathbb{D}^{\text {num }}$ and shown, as a function of $w$, in Figure 12. In the same figure, also the number of iterations required to reach a set error level is plotted.

This figure shows that smoothing $\sigma$ only marginally decreases $R$ while the number of iterations required to solve the problem increases. The increase in number of iterations can be explained by the fact that $\sigma$ is increased so that the smooth PML contrast function achieves the same attenuation as the step function PML contrast function, and thus that more contrast is present.

\section{E. Complex PML Contrast Function}

Evanescent and attenuative waves were shown in subsection II-B to be unaffected by PMLs if $\sigma(\vec{r})$ is real valued. Even though such waves are attenuated without the need for PMLs, in this experiment we try to increase their attenuation by applying PMLs with complex $\sigma^{\prime}(\vec{r})=(1-i \alpha) \sigma(\vec{r})$. For $\sigma(\vec{r})$ a step function is used.

The modelled situation is that of Figure 6, and simulations are performed at a frequency of $10 \mathrm{MHz}$. The real part of the PML contrast amplitude is fixed at $\sigma=50 \mathrm{MHz}$, a value

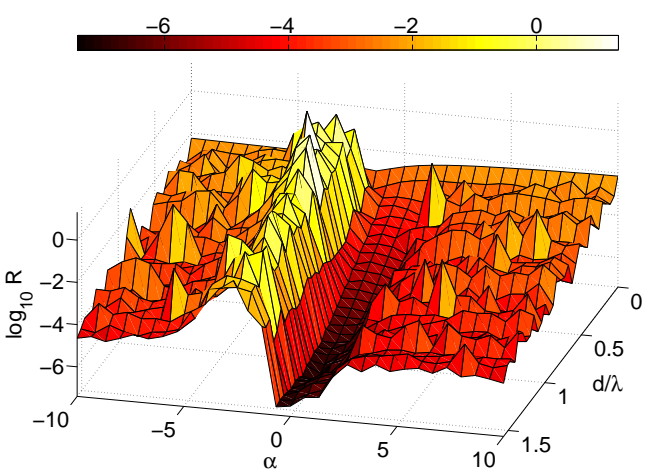

Fig. 13. Normalized sum of scatter pressure field $\hat{p}^{\text {scat }}$ at $\partial \mathbb{D}^{\text {num }}$ as a function of $\frac{d}{\lambda}$ and $\alpha$ after applying a PML with complex contrast function $(\sigma=50 \mathrm{MHz}$ ) for attenuative waves. Simulations are performed in the domain depicted in Figure 6 at a frequency of $10 \mathrm{MHz}$. The figure shows that strong attenuation is achieved for a real valued contrast function. Color version can be found online. Media-color 13

found in previous experiments to yield strong damping of propagating waves, and $\alpha$ and $\frac{d}{\lambda}$ are varied. The source is a transducer with a radiating surface of $8 \times 8 \mu \mathrm{m}^{2}$.

The attenuative waves are generated by using complex medium parameters for the background medium, mathematically represented by taking the wavenumber $\hat{k}$ in the Green's function complex and equal to $\hat{k}(1+i \beta)$. In this experiment, $\beta=0.1$ which amounts to much stronger attenuation than typically found in biomedical tissue. This ensures that any effects found here will be much less pronounced in practical biomedical applications.

The normalized sum of Equation (21) is computed over $\partial \mathbb{D}^{\text {num }}$ and shown, as a function of $\alpha$ and $\frac{d}{\lambda}$, in Figure 13. It is clear from this figure that strong damping is achieved for a purely real $\sigma^{\prime}(\vec{r})=\sigma(\vec{r})$ as the remaining scatter pressure field at $\partial \mathbb{D}^{\text {num }}$ is low(est) in that case.

In experiments not treated here it was found that convergence for all $\alpha \neq 0$ was much slower than for $\alpha=0$. This is explained by the fact that allowing $\sigma(x)$ to become complex introduces more contrast, see Equation (14), similar to increasing the magnitude of $\sigma$.

In subsection II-B it was predicted that allowing for a complex $\sigma(\vec{r})$ might lead to more energy being reflected off $\partial \mathbb{D}^{\mathrm{PML}}$. In the final experiment we use the configuration depicted in Figure 8 to compute, in the case of attenuative waves, the ratio $R$ of Equation (22) by summing the scattered field over $\mathbb{D}^{\text {num }}$ for various $\alpha$ and $\frac{d}{\lambda}$, again at a frequency of $10 \mathrm{MHz}$. The results are shown in Figure 14.

It is obvious from this figure that indeed for all $\alpha \neq 0$ the energy reflected off the PML is larger than for $\alpha=0$. Thus, considering damping, reflectionlessness and convergence rate, the best results are obtained by using a purely real $\sigma(\vec{r})$.

\section{CONCLUSION}

In this paper, Perfectly Matched Layers (PMLs) have been applied to frequency domain acoustic scattering integral equation problems. It is demonstrated that strong attenuation (a factor of 200 in amplitude) of scatter pressure fields is achieved in 


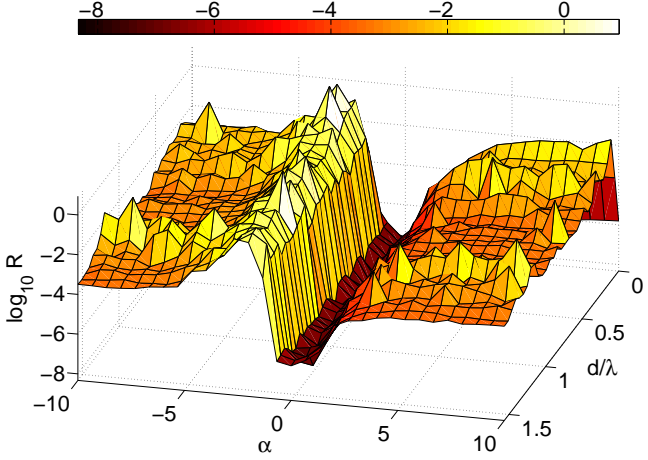

Fig. 14. Normalized sum of scatter pressure field $\hat{p}^{\text {scat }}$ over $\mathbb{D}^{\text {num }}$ as a function of $\frac{d}{\lambda}$ and $\alpha$. Simulations are performed for attenuative waves in the domain depicted in Figure 8 at a frequency of $10 \mathrm{MHz}$. The figure shows that the smallest reflection off the PMLs, with complex contrast function $(\sigma=50 \mathrm{MHz}$ ), is achieved for a real valued contrast function. Color version can be found online. Media-color 14

layers with a thickness of less than a wavelength, and that the PMLs themselves are virtually reflectionless. Thus, additional reflections introduced by truncating the computational domain in the presence of contrast at the domain boundary can effectively be suppressed by PMLs.

In this work it is also shown that the frequency domain integral equation method, both with and without PMLs, accurately reproduces pressure fields by comparing results to analytical solutions. We have shown and explained that applying PMLs deteriorates the convergence of the applied inversion scheme, and that this deterioration is strongly frequency dependent.

Furthermore, we have shown that using a smooth varying PML contrast function yields similar amplitudes for reflections off the PML as compared to a step function, and that convergence of the iterative scheme is faster using a step function. The slight reduction of the reflection generated by the PML does not justify the cost of a lower convergence rate, hence we conclude that using a step function for the PML is sufficient.

Finally we found that allowing a complex valued PML contrast function did not further reduce attenuative waves, whereas convergence is slower and more energy reflects off the PML itself. This implies that a purely real valued PML contrast function should be used.

\section{REFERENCES}

[1] JA Jensen. Field: A program for simulating ultrasound systems. volume 4, page 351353. Citeseer, 1996. 10th NordicBaltic Conference on Biomedical Imaging.

[2] EJ Alles and KWA van Dongen. Simulation of large spatial domain ultrasound scattering problems. Proceedings of NAG/DAGA 2009 Rotterdam, pages 328-331, 2009.

[3] KJ Baumeister, W Eversman, JW White, and RJ Astley. Acoustics in variable area duct- finite element and finite difference comparisons to experiment. AIAA Journal, 21:193-199, 1983.

[4] JT Hunt, MR Knittel, CS Nichols, and D Barach. Finite- element approach to acoustic scattering from elastic structures. The Journal of the Acoustical Society of America, 57:287, 1975.

[5] JT Fokkema and PM van den Berg. Seismic applications of acoustic reciprocity. Elsevier, Amsterdam, 1993.

[6] KWA Van Dongen, C Brennan, and W Wright. A reduced forward operator for acoustic scattering problems. pages 294-299. Institution of Electrical Engineers, 2005. IEE conference publication.

[7] KWA Van Dongen and WMD Wright. A forward model and conjugate gradient inversion technique for low-frequency ultrasonic imaging. The Journal of the Acoustical Society of America, 120:2086, 2006.

[8] B Engquist and A Majda. Absorbing boundary conditions for the numerical simulation of waves. Mathematics of Computation, 31(139):629651, 1977.

[9] JP Brenger. A perfectly matched layer for the absorption of electromagnetic waves. Journal of computational physics, 114(2):185-200, 1994.

[10] WC Chew and WH Weedon. A 3d perfectly matched medium from modified maxwell's equations with stretched coordinates. Microwave and Optical Technology Letters, 7(13):599-604, 1994.

[11] JP Brenger. Three-dimensional perfectly matched layer for the absorption of electromagnetic waves. Journal of computational physics, 127(2):363-379, 1996.

[12] FD Hastings, JB Schneider, and SL Broschat. Application of the perfectly matched layer (pml) absorbing boundary condition to elastic wave propagation. Journal of the acoustical society of America, 100(5):3061-3069, 1996.

[13] HW Chew and QH Liu. Perfectly matched layers for elastodynamics: A new absorbing boundary condition. Journal of computational acoustics, 4:341-359, 1996

[14] WC Chew and QH Liu. Using perfectly matched layers for elastodynamics. pages 366-369, 1996. Antennas and Propagation Society International Symposium, 1996. AP-S. Digest.

[15] QH Liu and J Tao. The perfectly matched layer for acoustic waves in absorptive media. The Journal of the Acoustical Society of America, 102:2072, 1997.

[16] X Yuan, D Borup, JW Wiskin, M Berggren, R Eidens, and SA Johnson. Formulation and validation of berenger's pml absorbing boundary for the fdtd simulation of acoustic scattering. IEEE transactions on ultrasonics, ferroelectrics, and frequency control, 44(4):816-822, 1997.

[17] SG Johnson. Notes on perfectly matched layers (pmls), 2008

[18] Wolfram website on Analytic Continuation. http://mathworld.wolfram. com/analyticcontinuation.html, 2009.

[19] HA Van der Vorst. Bi-cgstab: A fast and smoothly converging variant of bi-cg for the solution of nonsymmetric linear systems. SIAM Journal on scientific and Statistical Computing, 13:631, 1992.

[20] P Zwamborn and M van den Berg. The three-dimensional weak form of the conjugate gradient $\mathrm{fft}$ method for solving scattering problems. IEEE Transactions on Microwave Theory and Techniques, 40(9):1757, 1992.

[21] WC Elmore and MA Heald. Physics of waves. Dover Publications, New York, 1985.

[22] W Thompson Jr. Radiation from a spherical acoustic source near a scattering sphere. The Journal of the Acoustical Society of America, 60(4):781-787, 1976. 